\title{
Deterministic Mathematical Modelling of Platform Performance Degradation in Cyclic Operation Regimes
}

\author{
Nenad Kapor ${ }^{1}$ - Momcilo Milinovic ${ }^{1}-$ Olivera Jeremic ${ }^{1}$ - Dalibor Petrovic ${ }^{2}$ \\ 1University of Belgrade, Faculty of Mechanical Engineering, Serbia \\ 2University of Defense, Military Academy, Serbia
}

\begin{abstract}
This paper considers the modelling of extreme-capability working platforms that are operated in periodic cycles, each cycle having a predefined number of operations that affect the working surfaces. A novel hypothesis is introduced about the platform-degrading effects that cause an equivalent decrease in the successful operations after repeated cycles. Deterministic modelling, based on the basic equations of Lanchester and Dinner, is generalized here to include coupling between parameters. The newly developed mathematical model of performance degradation is in good agreement with both experimental measurements and numerical simulations. It is assumed that the new variables and their correlations link the Gaussian distribution and the observed performances of the testing platforms. Relative probability dispersions of affected surfaces are derived, as a new indirect referencing figure of merit, to describe simulations and compare them to experimental test data. The model proves a hypothesis that the degrading effects are a function of the platform capacity, frequency of operations and the number of available cycles. Degradation effects are taken into account through an equivalent decrease of effective operation capacities, reflected on the properties of the affected operating surfaces. The obtained estimations of degradation could be used in the planning of platform capacity as well as in the selection of real affected surfaces in various machining systems and for a wide range of different materials. Keywords: cycles, operations, extreme machine platforms, probabilities, deterministic modelling
\end{abstract}

Highlights

- Modelling of extreme-capability working platforms that are operated in periodic cycles.

- Proposed methodology for performances degradation caused by operations composed in cycles.

- Using Gaussian probability distribution law to predict degradation measures.

- Predicting the changes of probability dispersion based on modelling and experimental data.

- Determination of an analytical model based on a hypothesis that the degrading effects are a function of the platform capacity, frequency of operations and the number of available cycles.

\section{INTRODUCTION}

Machines operating in cycles and their properties have not been studied in depth in literature and, as such, are not well described by integral mathematical models. If the effects of their operation are actions on the given working surfaces under given constraints, then the quality of the affected surfaces can be described by reliability functions. In this manner, the operating capabilities of the platform can be determined. A majority of the published papers use a standard approach to the measured performances that depend on the machine's designed purposes. Such processes are described in [1] to [3] for the abrasive flow machines (AFM) with which material is hardened by randomly treating the working surface with abrasive particles with polymeric fillers, and dispersed within the flow media. The authors of [1] classified the work piece parameters into three groups based, among others, on the number of cycles (operations) and the machining time. Some of these parameters were determined experimentally in [2], in which the authors recognized that the parameters denoted as the creeping time and the cycles frequency have impact on the quality of the machining process. In [3], the authors experimentally prove that the aforementioned parameters influence the process. Common for all three papers is that they do not include hidden random effects caused by particles affecting the surfaces in cyclic operations, although such effects significantly influence the quality of the surface treatment. In all three papers, there is no mathematical modelling of the process.

Another similar type of machine with cyclic operation affecting working surfaces is described in [4] as shot-peening (SP) platforms. They bombard a surface with spherical beads to increase the material fatigue strength. The physical modelling of the influence of the bead shapes on the performance of the surface hardening process is presented in [5]. Random surface effects due to bombing cycles are a result of the quality of the machine's performance. However, the connection between the effects and the particular operations is missing in [5]. Paper [6] utilizes a risk function to consider the example of solar rays hitting a surface as a random process. In fact, the determination of the risk function dumping requires much more precise estimations of the distribution if probabilities 
of the effect occurrence on the attacked surface. However, this paper lacks a mathematical model of random disturbances of these probabilities. Common for the description of the processes in both AFM and SP machines, as well as the processes described in [6], is that they lack the deterministic or probabilistic mathematical modelling of cycles and their parameters on the efficiency of the final process.

Mechanical engineering of extreme machines in defence technologies have particular operations grouped in the cycle regimes. These operations affect the working surfaces or areas, with constrained machine capacities with regard to operation numbers. The modelling of efficiencies in such cases is usually done using the deterministic differential theories of operational research. This approach is based on the so-called Lanchester and/or Dinner equations of particular probabilities and their distribution laws, as presented in detail in [7], as well as in [8]. Their equations use variable attrition rates as the frequencies in operations probabilities, similar to [9], in which surface point effects are taken with variable probabilities. The modelling of cycles' efficiencies in these references is performed with coupled equations, in which two subjects simultaneously affect each other. Their actions are interdependent but different; their efficiencies also evolve differently over time. This approach is not entirely useful to define a standalone efficiency estimation for a single subject.

A mathematical model of the equipment with constrained capacities that generates identical repeated operations in a given order is presented in [10] for air platform equipment. The main contribution of this paper is the treatment of the action on the working surfaces as a random process, but the probability distribution laws on the affected surfaces are missing. The two-dimensional Gaussian distribution laws, used for welding processes, as referred in [11], could be useful in the estimation of random processes on surfaces.

According to the state-of-the-art analysis as presented in the cited papers, there is no comprehensive mathematical model, proven by experimental data, that would be capable of explaining relations between the machine's cyclic performances, its capacities and the quality of randomly affected surfaces, as well as the designed processing time. This is because the papers do not consider two repeating processes simultaneously acting on a single object (one as working and the second as redundant or parasitic), which together change the quality of the expected performances.
The objective of this paper was to develop a general joint mathematical model that includes all pertinent factor that influence the final efficiency of processing, thus enabling the simulation and evaluation of these parallel processes.

Based on the specific requirements for sequential processing of the surfaces, a mathematical model is developed using a deterministic approach treating the surface processes as random variables.

The objective was to test the efficiency of cyclic operations affecting the working surfaces, essentially by considering differences caused by the capacities and operation rates of the processing machine. This was shown using the experimental data on operation platforms with extreme performances.

The novelty of the approach presented in this manuscript is the redesign of coupled deterministic equations done in a new manner. In the literature above, these equations are employed to describe the mutual effects of objects as a function of the elapsed process time. This approach in the literature makes the time functions dependent on the performances of two objects. In our approach, one object executes two operations in parallel, one of them comprising the working process itself, and the other, parasitic, occurring as self-degradation dependent on the first one. Both happen on the same object, i.e. the operation platform performing the same action. The new approach composes deterministic equations to describe this and to measure changes in the platform efficiency. With our approach, the quality of the working process is the convolution of both kinds of operations in one cycle, as well as their frequencies. The number of cycles influences the random arguments and reflects the probabilities of working surface coverage that follow the two-dimensional Gaussian distribution laws. This was taken as the measure of the changes in quality due to self-degradation.

\section{EXPLANATION OF THE GENERAL MODEL}

The model offers the possibility of evaluating the degradation of the platform performance, with regards to the equipment and devices contained within the platform. The quality of the affected surface is regarded as the dimension of probability dispersion. This dimension appears during the execution as the consequence of cycle duration and the operations frequency, as well as of the capacities of the platform. The approach presented in [12], which developed operations frequency coupled with execution probabilities as the combined attrition parameter, was used in developing our general model. Changes 
of probability dispersions of random values on the affected surface appear in the form of the Gaussian distribution law. The degradation of the platform properties through the operation cycles is represented by changes in the Gaussian distribution. This is valid under the assumption that one particular Gaussian distribution function is valid for each cycle in the working regime.

In our approach, this function is distributed in successive cycles in the form of extended probability dispersions of both random arguments in the two surface directions. Consequently, the changing of efficiencies over time is measured by the resulting effects on the new randomly affected surfaces. The decrease in the efficiency with each new cycle in reflected in the new less-affected surfaces. This also results in the degradation of the working platforms' capabilities caused by less effective particular operations in the cycles. The cause of this degradation could be a consequence of rapid highenergy operations realized in short-time sequences (high mechanical power values) in successive, orderly repeated cycles, similar to those described in paper [13]. However, according to that paper, the affected points on the surfaces do not obey any probabilistic law, and thus there is no error distribution as a modelling parameter. In our research, we use the changes of the probability dispersion (PD) after each cycle, due to all the errors in the cycle, as a measure of the platform efficiency. These changes are caused by the generator of the cycles and by its self-degradation, and are reflected in the decreased number of declared operations. This makes the designed operational capacities of the platforms less effective with the number of cycles.

In order to estimate the degraded platform performances by means of time-based simulation, new relative parameters have been accepted in the modelling.

The deterministic modelling of the estimations of the so-called vulnerability performances is presented in [14] and [15]. The performances considered there are similar to our degrading platforms' performances. The models presented in [16], called Pexpot, Levpot and Dynpot, were also developed as vulnerability considerations based on the attrition rate function and thus indirectly describe the kind of expected degradation capabilities. An essential difference of our model is that the degradation of the system appears as a direct consequence of self-degradation caused by the effects of the repeated cycles. The designed frequencies and functional probabilities, contained in each operation, are reflected in the full platform capacity on the affected surfaces. This effect makes the proposed model more useful in planning the redesigning of platform capacities for required affected surfaces.

\section{MATHEMATICAL MODELS}

In the presented model, the platform has the capacity of $M_{p_{0}}$ particular operations oriented toward the working surface. These operations occur in dynamic regimes with successive frequencies $\lambda$ and a probability of surface action of approximately $p=0.997$. This is provided using the maximum technical dimensions of the surface, which correspond to the $64 \mathrm{PD}_{\mathrm{av}}{ }^{2}$. The width and depth of the surface used eight of the same average probability dispersions PDav, in both surface directions. Average probability dispersions $\mathrm{PD}_{\mathrm{av}}$ are taken as an equal of the expected Gaussian distribution of two-dimensional random arguments. The probability variations are represented as functions of the cycle number and of the full capacity of operations. The designed properties of these processes are consequently the function of probability changes. The adopted hypothesis is that the degradation of the platform performances is an imaginary effect, able to be explained by the values of the effective and ineffective numbers of operations. This ensures a possibility of considering the ineffective number to be a value increasing with the number of cycles during the exploitation time. In that sense, the increasing number of ineffective operations corresponds to the increase of cycle probabilities dispersions.

Operative consumption is realized in cycles with the same sequential probabilities of operations, $p$, as in [10]. In that case, the frequency of executions of real operations, as the real rate of operation, is:

$$
\frac{d M_{p}}{d t}=-\lambda p=-\alpha_{p}
$$

This determines the remaining number of operations as the $M_{p}=M_{p}(t)$ in each moment of time $t$ in the cycle duration interval.

It is expected that the probability would not have a fixed value but would vary over exploitation time. The changes of probability function $p$ could mean random changeable performances that disturb the rate of real operations on the working surfaces. The changes in probabilities affect the rate of real operations $\dot{M}_{p}$ in Eq. (1). This is not really possible because the frequency of operation executions is a designed property of the platform hardware. The present hypothesis has only an imaginary effect. 
The acceptable solution could be to recalculate the influence of the number of ineffective operations on the new probable dispersion PD reflected in a new Gaussian distribution but for the unchanged execution operations probabilities. The consequence is that the model has to consider the extended working surfaces, with new dimensions $64 \mathrm{PD}^{2}$ engaged in operations after each cycle.

The platform degradation, as an imaginary effect, is a process in the real cycle time and is simultaneously parasitic in real operations. A new value of the modified equivalent number of operations $m_{p}(t)$ is diminished by this imaginary effect. This is generated as a current and recalculated capability of working platform. The new value is lower than the real number of the remaining operations $M_{p}(t)$. At the very beginning, it is equal to the real available capacity $m_{p_{0}}=M_{p_{0}}$.

The reason is underpinned by the fact that the model of self-degradation is viewed as a new, fictive rate of equivalent non-effective operations changes $\dot{m}_{p}$, which is not equal to the rate of real operation $\dot{M}_{p}$. This orients the mathematical model to consider the share of degraded value on each of the real operations and, by that effect, redesign the remaining number of operations available on the platform. Such a transformation implies that the degrading rate of $\dot{m}_{p}$ and the real rate $\dot{M}_{p}$ during each cycle are proportional to the remaining equivalent dimensionless number of operations $1 / m_{p}$. The correction coefficient is the portion of one operation within the actual remaining equivalent number $m_{p}$.

Based on the previous concept, the differential equation for the degradation rates, Eq. (1), becomes:

$$
\frac{d m_{p}}{d t}=-\alpha_{p} \frac{1}{m_{p}}
$$

Since the model of equivalent numbers is a function of time and the current equivalent number $m_{p}$, as the instantaneous remaining capacity, the platform performances are degraded continually with each cycle. This always means a new valid number of operations with regard to the remaining capacity. It is inappropriate to use the approach as constant and fixed for any platform capacity since it is dependent on the available number of operations. The relative degradation of the platform capacity is taken as more acceptable in modelling with the functional ratio $\mu_{p}(t)=m_{p}(t) / m_{p_{0}}$ where $\mu_{p}(t=0)=1$, as the current relative capacity degradation of the platform. The general differential equation (Eq. (2)) of new functional $\mu_{p}$, by methodology given in [7], is:

$$
\frac{d \mu_{p}}{d t}=-\alpha_{p} \frac{1}{\mu_{p} M_{p_{0}}^{2}} .
$$

If the platform, under the same conditions, executes repeating working cycles $n$ times, in equal time intervals for each cycle of $\Delta t$, and the rate of operations in the cycle is the same, then using Eq. (3), any of $i$ cycles (where $i=1,2, \ldots, n$ ) is used at the beginning of a new, redesigned equivalent number of operations from the previous cycle. The current relative degrading in cycle is defined as a new differential equation:

$$
\frac{d \mu_{p_{i}}}{d t}=-\alpha_{p} \frac{1}{\mu_{p_{i}} m_{p_{(i-1)}}^{2}}, \quad i=1,2,3 .
$$

The solution of Eq. (4) is:

$$
\mu_{p_{i}}(t)=\sqrt{1-\frac{2 \alpha_{p}}{m_{p_{(i-1)}}^{2}} t} .
$$

The function of the current relative capacity degradation of the platform full capacity after $(i-1)$, and during the $i^{\text {th }}$ cycle at an instant $(i-1) \Delta t<t<i \Delta t$, similarly to [10], is:

$$
\mu_{p}(t)=\mu_{p_{i}}(t) \prod_{j=1}^{i-1} \mu_{p_{(j)}},
$$

with the condition $\prod_{j=1}^{i-1} \mu_{p_{(j)}}$ for the $i=1$. The estimation of the relative efficiency of the current process is the function of the affected and the initial working surface. This functional is determined for the unaffected, remaining surface at each cycle and the final working surface from the previous cycle, taken as initial in the current one. It is given in the form:

$$
\mu_{p_{i}}(t)=1-\frac{S_{i}(t)}{S_{(i-1)}^{c}}
$$

The differential equation of the relative efficiency of the current process, as the remained relative surface within operation cycle considered as the degraded ones, according to a similar differential equation in [7] and [10], is:

$$
\frac{d \mu_{c_{i}}}{d t}=-U_{i} \mu_{p_{i}} \mu_{c_{i}}, \quad 0<t<\Delta t
$$

If the platform operates in cycles without degradation effect, its $\mu_{p_{i}}=1$, the functional $\mu_{p_{i}}$ 
does not affect Eq. (8) and the coupling of Eqs. (4) and (8) is lost. Consequently, the relative efficiency of the current process, denoted by $\mu_{c_{i}}^{*}$, during the undegrading surface processing in the cycles is described by:

$$
\frac{d \mu_{c_{i}}^{*}}{d t}=-U_{i} \mu_{c_{i}}^{*}
$$

In both Eqs. (8) and (9), the operation number in one cycle is the designed capability, and could be variable. This depends on the designed cartridge capacity used for continual operations in the short impulse regimes. The well-balanced example between the number of operations and the covered affected surface in one cycle is the referent platform given in [10]. It uses cartridges of maximum $N_{p}(\Delta t)=8$, and its cycle expires in 4.4 seconds. The accepted functional designed capability of the platform, redesigned for the considered example, is:

$$
U_{i}=\lambda p \frac{1.82 \sigma}{S_{o}} .
$$

Appropriate values need to be calculated for each platform cartridge with their declared performances regarding the expected affected surface. The solution of Eq. (8) is:

$$
\mu_{c_{i}}(t)=\mu_{c_{i}}(0) e^{\frac{U_{\mathrm{i} m_{p(i-1)}^{2}}^{2}}{3 \alpha_{p}}\left(\mu_{p_{i}}^{3}-1\right)},
$$

while for the ideal, un-degraded effect, from Eq (9), it takes the form:

$$
\mu_{c_{i}}^{*}(t)=\mu_{c_{i}}^{*}(0) e^{-U_{i} t}
$$

The next appropriate assumptions for the initial conditions are used:

- Model A with initial conditions for the relative efficiency of the current process at the very beginning $\mu_{c_{i}}(0)=1-\frac{S_{i}(0)}{S_{(i-1)}^{c}}=1$.

- Model B with variable relative efficiency of the current process at the very beginning $\mu_{c_{i}}(t=0)=\mu_{c_{i}(i-1)}<1$ taken in the next cycle from the end of the previous one. In both cases, the designed cycle capability of the platform $U_{i \max }=\lambda p \frac{1.82 \sigma}{S_{o}}=$ const and is constant in all cycles as a declared value.
The cumulative relative efficiency of all finished processes is:

$$
\mu_{c}=\prod_{i=1}^{n} \mu_{c_{(i)}} .
$$

For the experimental verification of the correlation between the platform capacities and the affected surfaces for the degraded as well as for the un-degraded (available) number of operations, new expressions were required. If all available cycles on the platform are expired, the full affected surface $S_{p}$ and the working surface $S_{0}$ can be correlated. The correlation could be expressed via a relation analogous to Eq. (7), using degradation effects on the surface given by cumulative relative efficiency of all finished processes in Eq. (13). This yields the relation

$$
S_{0}=\frac{S_{p}}{1-\mu_{c}} \text {. }
$$

The same logic, analogous to Eq. (14), could be used for the non-degraded working surface $S_{0}^{*}$ and the affected surface $S_{p}^{*}$ equations. They also have to be related to the cumulative relative efficiency of all finished processes without degradation of $\mu_{c}^{*}$, which is a product similar to Eq. (13), with $\mu_{c(i)}^{*}$ determined from Eq. (12), in the same form as Eq. (14). For the ideal designed number of operations, as well as for the degraded number, the particular affected surfaces are equal $\left(S_{p}^{*}=S_{p}\right)$. This is because no operation in the cycle is missed but is only exposed somewhere on the larger area. The working surfaces $S_{0}$ and $S^{*}{ }_{0}$ could be determined as the squares of the appropriate Gaussian linear values of average probability dispersion $\mathrm{PD}_{\mathrm{av}}$ for both cases. Since the degraded and the nondegraded cumulative relative efficiency of all finished processes are the functionally correlated to the surfaces given by Eq. (14), the dispersion values also satisfy these correlations in both cases.

Since the surfaces are taken as $8 \mathrm{PD}_{\mathrm{av}} \times 8 \mathrm{PD}_{\mathrm{av}}$, using basic Eq. (14) and their analogues, the ratio of PD* in un-degraded and PD of degraded cases is expressed by:

$$
\frac{P D^{*}}{P D}=\sqrt{\frac{1-\mu_{c}}{1-\mu_{c}^{*}}} .
$$

This new approach provides the method for a comparison of degraded and un-degraded surfaces of accepted dimensions, or for treating expected degrading by constraining the allowable ratio. 


\section{SIMULATION DATA AND RESULTS}

Simulation tests are realized using MATLAB software package and compared with experimental research. The basic assumptions in the simulations were:

- Platform capacities and cycles performances presented in Table 1 are used for simulation testing, as well as in experimental modelling. These data are used in the platform degradation modelling;

- Numerical test is provided for the 8, 16, 24, 32, and 40 operation capacity of the platform. As it was accepted in the mathematical model, one cycle had 8 operations and expired in a nominal time of 4.4 seconds.

Fig. 1 represents the simulation results of the current relative degradation of successive ordering operations in the sequential cycles. The degraded values are positive and decreasing, bounded with value 1 from the upper side. The simulation shows that at the end of each cycle, the current relative degradation final value is decreasing, regardless of the number of operations remaining constant.

The consequence of the model is that platform capability degrades more rapidly in each subsequent cycle, regardless of the same number of operations. It is the consequence of the decreasing number of equivalent operations after each real execution rate. The rapidest decrease of the current relative capacity degrading has been observed for the platform with 8 designed operations within one cycle.

Lower degradation occurs on platforms that have $16,24,32$ and 40 operations as the full capacity.

Platforms with higher initial capacities, e.g. the number of operations, have smaller gradients and lower degradation at the end of the cycle.

Fig. 2 represents the equivalent number of degraded operations starting with a different real number of platform capacities. It is visible that the gradient disperses with time. This process shows the minimum gradient for the platforms tested with 40 or more real operations compared to platforms with fewer capacities used in the simulation model.

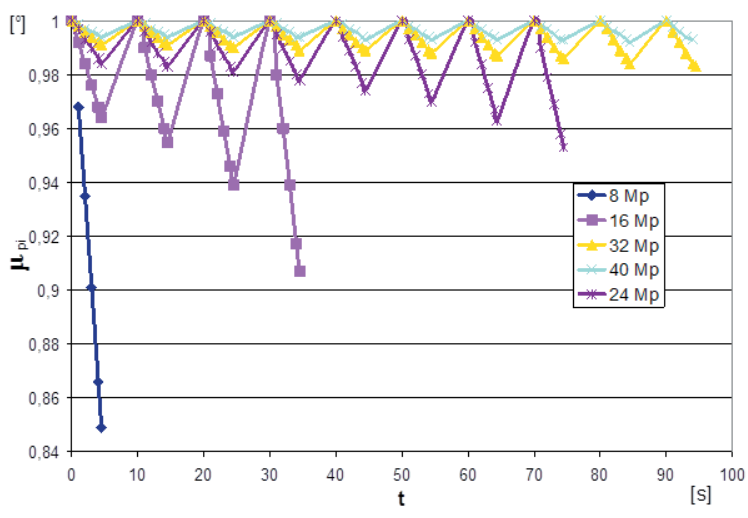

Fig. 1. Functions of current relative degradation of successive ordering operations in the sequential cycles

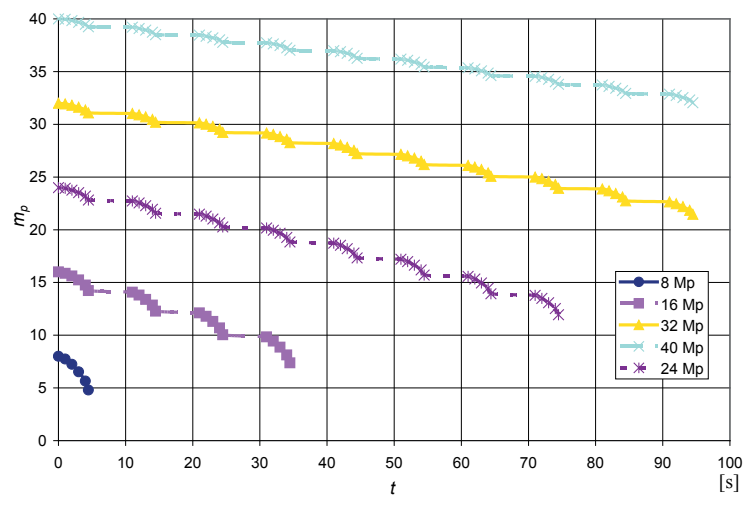

Fig. 2. Real initial and degraded equivalent number of operations on the platforms with different operation capacities

Fig. 3 explains the model of cumulative relative efficiency of all finished processes. As it shown in Figs. 3a and b, the unaffected surface decreases with the increasing number of operations. This decrease is more or less similar to the experimental results of surface roughness decrease, presented experimentally in [1]. This paper has shown similar surface effects as our model.

Table 1. Simulated platforms performances

\begin{tabular}{ccccccccc}
\hline $\begin{array}{c}\text { Capacity } \\
\text { variant }\end{array}$ & $\begin{array}{c}\text { Number of } \\
\text { operations } \\
\text { in one tool set }\end{array}$ & $\begin{array}{c}\text { The number } \\
\text { of tool-sets }\end{array}$ & $\begin{array}{c}\text { Available number } \\
\text { of platforms } \\
\text { operations }\end{array}$ & $\begin{array}{c}\text { Rate of } \\
\text { operation } \lambda \\
{[1 / \mathrm{s}]}\end{array}$ & $\begin{array}{c}\text { Working } \\
\text { surface } \\
{\left[\mathrm{m}^{2}\right]}\end{array}$ & $\begin{array}{c}\text { Effective } \\
\text { tool radii } \\
R c[\mathrm{~m}]\end{array}$ & $\begin{array}{c}\text { Elementary } \\
\text { surface efficiency } \\
\sigma\left[\mathrm{m}^{2}\right]\end{array}$ & $\begin{array}{c}\text { Number of designed } \\
\text { affected operations } \\
\text { in one cycle }\end{array}$ \\
\hline 1 & 4 & 2 & 8 & 1.8 & 1.7 & 0.126 & 0.05 & 8 \\
\hline 2 & 4 & 4 & 16 & 1.8 & 1.7 & 0.126 & 0.05 & 8 \\
\hline 3 & 4 & 6 & 24 & 1.8 & 1.7 & 0.126 & 0.05 & 8 \\
\hline 4 & 4 & 8 & 32 & 1.8 & 1.7 & 0.126 & 0.05 & 8 \\
\hline 5 & 5 & 8 & 40 & 1.8 & 1.7 & 0.126 & 0.05 & 8 \\
\hline
\end{tabular}


Paper [1] also reported significant changes in the first 20 operations (so-called cycles in [1] and [2]) and the gradual reaching of a saturated level. The result shown in Fig. 1 also shows the saturation performance of relative degrading, but with at least 40 operations. It could be seen that those values are of the same order of magnitude.

Experiments in [1], developed for surface roughness, have shown that the roughness decreased slightly after 50 operating cycles. Comparative considerations about similarities of surfaces affecting processes, shown in Fig. 3, regardless of whether they have been tested on the different machines with different purposes, showed the same sort of behaviour.

Two models of surfaces with different initial conditions marked as a) and b) shown in Fig. 3 are tested in simulation. The first tested case, for the both models, is degraded platform performance and the

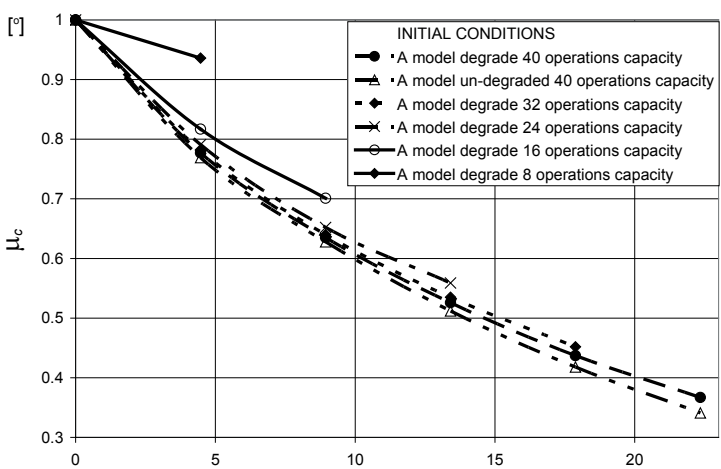

a)

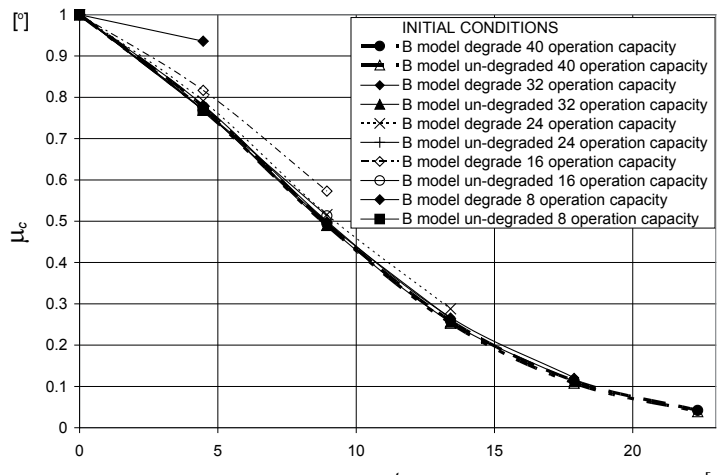

b)

Fig. 3. Current relative efficiency of affected surface with variable operation designed capacities and number of cycles; a) model $A$, and $b$ ) model $B$

Table 2a. Experimental data for the probability dispersions

\begin{tabular}{ccccc}
\hline Cycle No & $\begin{array}{c}\text { Model } \mathrm{B}: \\
\text { Experimental test of } \mathrm{PD}_{\mathrm{av}} \text { data }[\mathrm{m}]\end{array}$ & $\begin{array}{c}\text { Model B: } \\
\text { Dispersion error [\%] }\end{array}$ & $\begin{array}{c}\text { Model } \mathrm{A}: \\
\text { Experimental of } \mathrm{PD}_{\mathrm{av}} \text { data [m] }\end{array}$ & $\begin{array}{c}\text { Model A: } \\
\text { Dispersion error [\%] }\end{array}$ \\
\hline 1 & 7.5 & 36 & 5.0 & 32 \\
\hline 2 & 5.0 & 10 & 5.0 & 32 \\
\hline 3 & 5.0 & 10 & 9.0 & 22 \\
\hline 4 & 5.0 & 10 & 9.0 & 22 \\
\hline 5 & 5.0 & 10 & 9.0 & 22 \\
\hline 6 & 5.5 & 0 & 7.5 & 1 \\
\hline $\mathrm{PD}_{\mathrm{av}}$ & 5.5 & 12.67 & 7.4 & 21.8 \\
\hline
\end{tabular}

Table 2b. Simulation of the probability dispersions

\begin{tabular}{|c|c|c|c|c|c|c|c|}
\hline $\begin{array}{l}\text { Total cycles } \\
\text { time t [s] }\end{array}$ & $\begin{array}{c}\text { Degraded } \\
\mu_{c}\left[^{\circ}\right]\end{array}$ & $\begin{array}{c}\text { Non-degraded } \\
\mu_{c}{ }^{*}{ }^{\left.{ }^{\circ}\right]}\end{array}$ & $1-\mu_{c}\left[^{\circ}\right]$ & $\left.1-\mu_{c}{ }^{*}{ }^{\circ}\right]$ & $\mathrm{PD}_{\mathrm{av}}{ }^{*} / \mathrm{PD}_{\mathrm{av}}$ & $\begin{array}{l}\% \text { of surface } \\
\text { extension }\end{array}$ & $\begin{array}{l}\text { Operation } \\
\text { capacity }\end{array}$ \\
\hline$\mu_{c} \neq 1$ & & & & Model B & & & \\
\hline 4.47 & 0.936 & 0.769 & 0.064 & 0.231 & 0.526361 & 47.36 & 8 \\
\hline 8.94 & 0.468141 & 0.394497 & 0.531859 & 0.605503 & 0.937217 & 8.675 & 16 \\
\hline 13.41 & 0.117549 & 0.100695936 & 0.882451 & 0.899304 & 0.990586 & 2.84 & 24 \\
\hline 17.88 & 0.012616 & 0.010803896 & 0.987384 & 0.989196 & 0.999084 & 1.217 & 32 \\
\hline 22.35 & 0.000476 & 0.000401542 & 0.999524 & 0.999598 & 0.999963 & 0.608 & 40 \\
\hline$\mu_{c}=1$ & & & & Model A & & & \\
\hline 4.47 & 0.936 & 0.769 & 0.064 & 0.231 & 0.526361 & 47.36 & 8 \\
\hline 8.94 & 0.572717 & 0.482932 & 0.427283 & 0.517068 & 0.909042 & 10.67 & 16 \\
\hline 13.41 & 0.288294 & 0.247261184 & 0.711706 & 0.752739 & 0.972362 & 4.36 & 24 \\
\hline 17.88 & 0.120871 & 0.103355175 & 0.879129 & 0.896645 & 0.990184 & 2.4 & 32 \\
\hline 22.35 & 0.041622 & 0.035244115 & 0.958378 & 0.964756 & 0.996689 & 1.556 & 40 \\
\hline
\end{tabular}


second one is the non-degraded performance. Both curves of the cases, for both models, are shown in Figs. 3a and $\mathrm{b}$. The platforms with lower operation capacities, 8 and 16 , in simulations have greater differences for degraded and un-degraded tests for Model A. This is also valid in Model B. At higher operation capacities, 24, 32 and 40 have diminishing differences except in Model B where their differences disappear more rapidly than in Model A. The curves from Fig. 3 present the relative efficiency of the current process, with regard to cycle time as a continuously varying value.

Differences between the relative values of affected surfaces in the degraded and un-degraded cases have been recalculated on their new extended working surface dimensions and transformed into their probability dispersions ratios. The average probability dispersion, $\mathrm{PD}_{\mathrm{av}}$, as the measure of degraded surface is shown in experimental and simulation test data in Tables $2 \mathrm{a}$ and $\mathrm{b}$ from Eq. (15) for the platforms with different capacities and cycle numbers composed in the tool sets (Table 1). Repeated experimental tests have been realized in cycles with 8 operations in 6 realized experiments shown in Table $2 \mathrm{a}$. It is obvious that both experimental models, A and B, show strong degradation of the initial probability dispersions. The decrease is slowed down by the increasing number of operations in the new cycles. Saturation is achieved after 24 operations for Model A and after 16 operations for Model B.

It is obvious that Model $\mathrm{B}$, where the relative efficiency of the current process at the very beginning of cycles is taken from the previous cycle, has a smaller saturation decrease of $\mathrm{PD}_{\mathrm{av}}$ in both experimental and simulation cases.

In Table 2b, the dispersion in Model B is saturated after 24 operations from 47 to $2.84 \%$ and further approaches $1 \%$. In both models, after at least 40 operations, (in experiments after 48), the probability dispersion PD stopped decreasing and diminished.

The percentage of the errors measured by the surface extension over PD, for the model in simulations and in experiments, is about $4 \%$ for Model B and about 29\% for Model A.

This is because the decreased unaffected surface from the previous cycle in Model B is repeatedly taken as an initial surface for each next cycle. Consequently, errors increase as the cumulative values from the previous cycles. Taking into account the unsteadystate random processes on the designed platforms, predictions of efficiency made by this indirect modelling are of sufficiently quality.

\section{CONCLUSIONS}

In the present state of the art, in the field of surfaceaffecting machines, there is no unified model theory that connects the variable machine performances with effects on the affected surface determined in continuous working process. Operational research modelling offers some models of interaction of two interdependent objects. In our model, one object, a surface affecting machine as a platform operating in cycles, is self-degrading due to the effects of sequentially repeated cycles. In our hypothesis, it was assumed that the rate of degradation is variable and proportional to the rate of real operations divided by the actual remaining capacity. This novel approach is proved indirectly for the effects occurring on the working surface. These effects are measured by determining the statistical variations of the surface parameters.

The relevance of this model is that the real effects by the cyclic operations and also by the planned operation capacities could be predicted for the required surface effects. The model is also able to predict the extreme machine performances based on the expected quality for the surface treatment.

The presented simulation results are consistent with the experimental data and coincide with the research cited.

Further research in this area could involve an extension of the experimental data base in order to improve the simulation model according to the type of special extreme machines and their affected surfaces.

The model can be readily applied to additional processes and effects. This could be done by exponential redesign of the basic correlations between the rates of operations and the rates of degradations, or by including variable frequencies and probabilities.

\section{ACKNOWLEDGMENTS}

This paper is a part of the research within the Project III 47029 for MNES-RS in 2014-2015.

\section{NOMENCLATURE}
$M_{p}(t) \quad$ current number of operations,
$m_{p}(\mathrm{t}) \quad$ current number of degraded operations,
$\dot{M}_{p} \quad$ operation consumptions rate,
$\dot{m}_{p} \quad$ rate the fictive changes of non-effective operations(rate of equivalent operations),
$M_{p_{0}} \quad$ capacity of particular operations, 
$m_{p_{0}} \quad$ initial degraded number of operations,

$m_{p(i-1)} \quad$ initial degraded number from the previous cycle,

$N \quad$ number of cyclic operations,

$m_{p_{i}}(t)$ current number of operations during degradation,

$S_{0}^{*} \quad$ remaining non-degraded surface,

$S_{p} \quad$ affected surface

$S_{(i-1)} \quad$ remaining non-degraded surface after the $(i-1)^{\text {th }}$ cycle,

$S_{i}(t) \quad$ current non-degraded surface,

$\Delta t \quad$ cycle period,

$P D^{*} \quad$ probability dispersion of un-degraded, operations on the platform,

$P D \quad$ probability dispersion of working surface in degraded platforms operations,

$U_{i} \quad$ designed capability of the platform,

$\alpha_{p}=\lambda_{p}$ attrition rate of operations (consumption rate of operation numbers),

$p \quad$ probability of each operation,

$\mu_{p_{i}}(t)$ current relative degradation in the $i^{\text {th }}$ cycle

$\mu_{c_{j}}(t)$ relative efficiency of the current process,

$\lambda=-N_{p}(\Delta t) / t$ operation frequency for a given cycle,

$N_{p}(t) \quad$ number of operations per cycle,

$\sigma \quad$ elementary efficiency on the surface,

$\mu_{p}(t) \quad$ current relative capacity degradation,

$\mu_{c_{i}}^{*} \quad$ relative efficiency of the current process without degradation,

$\mu_{c_{i}(i-1)}$ relative efficiency of the current process, after $i-1$ cycles with degradation effect,

$\mu_{c} \quad$ cumulative relative efficiency of all finished processes.

\section{REFERENCES}

[1] Gov, K., Eyercioglu, O., Cakir V.M. (2013). Hardness effects on abrasive flow machining. Strojniški vestnik - Journal of Mechanical Engineering, vol. 59, no. 10, p. 626-631, DOI:10.5545/sv-jme.2013.1129.

[2] Kar, K.K., Ravikumar, N.L., Tailor, P.B., Ramkumar, J., Sathiyamoorthy, D. (2009). Performance evaluation and rheological characterization of newly developed butyl rubber based media for abrasive flow machining process. Journal of materials Processing Technology, vol. 209, no. 4, p. 22122221, D0I:10.1016/J.jmatprotec.2008.05.012.
[3] Rhoades, L. (1991). Abrasive flow machining: a case study. Journal of Materials Processing Technology, vol. 28, no. 1-2, p. 107-116, DOI:10.1016/0924-0136(91)90210-6.

[4] Zupanc, U., Grum, J. (2011). Surface integrity of shot peened aluminium alloy 7075-T651. Strojniški vestnik - Journal of Mechanical Engineering, vol. 57, no. 5, p. 379-384, DOl:10.5545/sv-jme.2010.142.

[5] Fang, L., Zhao, J., Li, B., Sun, K. (2009). Movement patterns of ellipsoidal particle in abrasive flow machining. Journal of Materials Processing Technology, vol. 209, no. 20, p. 60486056, DOI:10.1016/J.jmatprotec.2009.08.013.

[6] Lugarić, L., Majdandžić, Lj., Škrlec, D. (2010). Countrywide positioning of domestic solar water heating systems using risk analysis and geographical information system. Strojniški vesnik - Journal of Mechanical Engineering, vol. 56, no. 1, p. 3-17.

[7] Przemieniecki, J.S. (2000). Mathematical Methods in Defense Analyses, 3 rd edition. American Institute of Aeronautics and Astronautics, Redstone.

[8] Maybee, J.S. (1985). The theory of combined-arms lanchestertype models of warfare, Naval Research Logistic Quarterly, vol. 32, no. 2, p. 225-237, DOl:10.1002/nav.3800320204.

[9] Anderson, L.B. (1993). A heterogeneous shoot-look-shoot attrition process. Simulation and Gaming, vol. 24, no. 3, p. 277-293, DOI:10.1177/1046878193243001.

[10] Petrovic, D., Milinovic, M., Jeremic, O., Kovac, M., Kapor, N. (2014). Air force support operations and comparative performances. Symposium of Operational Research, p. 16-19. (in Serbian)

[11] Ghosh, A., Barman, N., Chattopadhyay, H., Hloch, S. (2013). A study of thermal behaviour during submerged arc welding. Strojniški vestnik - Journal of Mechanical Engineering, vol. 59, no. 5, p. 333-338, D0l:10.5545/sv-jme.2012.775.

[12] Scales, J.R. (1995). A modified lanchester linear process calibrated to historical data. Warfare Modelling, 3rd edition, Military Operations Research Society, Alexandria, p. 345-356.

[13] Milinovic, M., Jeremic, O., Kovac, M., Petrovic, D. (2014). Discrete deterministic modelling of autonomous missiles salvos. Defence Science Journal, vol. 64, no. 5, p. 471-476, DOl:10.14429/dsj.64.5791.

[14] Zipfel, P.H. (1995). Modelling and Simulation of Aerospace Vehicle Dynamics. American Institute of Aeronautics and Astronautics, Reston.

[15] Ball, R.E. (1985). The Fundamentals of Aircraft Combat Survivability: Analyses and Design. American Institute of Aeronautics and Astronautics, Redstone.

[16] Anderson, L.B., Miercort, F.A. (1995). On weapons scores and force strengths. Warfare Modelling, 3rd edition, Military Operations Research Society, Alexandria.

[17] Law, A.M., Kelton, W.D. (1982). Simulation Modelling and Analysis. McGraw-Hill, New York. 